RESEARCH ARTICLE

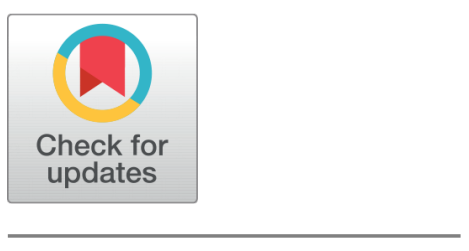

OPEN ACCESS

Received: 03.12 .2021

Accepted: 24.12 .2021

Published: 27.01.2022

Citation: Bajpai V, Kaur P, Kumar V, Basita A, Vasistha P, Kumar R (2022)

The Effect of Turbulence on Dynamic Radar Cross Section of Chaff Clouds. Indian Journal of Science and Technology 15(2): 62-68. https://doi.org/

10.17485/IJST/v15i2.2143

* Corresponding author.

prashantvasistha@dl.drdo.in

Funding: None

Competing Interests: None

Copyright: (c) 2022 Bajpai et al. This is an open access article distributed under the terms of the Creative Commons Attribution License, which permits unrestricted use, distribution, and reproduction in any medium, provided the original author and source are credited.

Published By Indian Society for Education and Environment (iSee)

ISSN

Print: 0974-6846

Electronic: 0974-5645

\section{The Effect of Turbulence on Dynamic Radar Cross Section of Chaff Clouds}

\author{
Vivek Bajpai ${ }^{1}$, Parneet Kaur ${ }^{2}$, Verandra Kumar ${ }^{3}$, Alok Basita ${ }^{4}$, \\ Prashant Vasistha ${ }^{*}$, Ravindra Kumar ${ }^{6}$ \\ 1 Technical Officer, Defence Laboratory, Jodhpur, India \\ 2 Sr Engr Jio Platform Ltd, Navi Mumbai, India \\ 3 Scientist D, Defence Laboratory, Jodhpur, India \\ 4 Scientist E, Defence Laboratory, Jodhpur, India \\ 5 Scientist G, Defence Laboratory, Jodhpur, India \\ 6 Scientist H, Defence Laboratory, Jodhpur, India
}

\section{Abstract}

Objective: This research reports the effect of turbulence on the dynamic Radar Cross Section (RCS) of chaff cloud. In the era of modern warfare technologies, most efficacious electronic countermeasure employed by the armed forces of various major powers is the Chaff. Chaff is based on the expulsion of numerous electrical dipoles of different length to cover the broad spectrum of radar frequency. This mechanism helps in deceiving the enemy missile by producing the required RCS. RCS of chaff cloud is a dynamic entity and rely on several aerodynamic factors. Methodology: Effects of turbulence on dynamic RCS are studied using Ansys Fluent for aerodynamic blooming simulation and Ansys HFSS for simulation of RCS of the bloomed chaff cloud. Findings: The subject of the paper corresponds to the significant effect of turbulence on the expeditious blooming of chaff cloud. The RCS of turbulent flow was higher by $1.5 \mathrm{dBsm}$ to $2.5 \mathrm{dBsm}$ for $50 \mathrm{msec}$. The turbulence effect has reduced the blooming time of chaff filaments in the range of $25 \mathrm{msec}-50 \mathrm{msec}$. Novelty: Turbulence plays important role in faster blooming of chaff cloud and achieving minimum threshold RCS required for deception of RF seeker missiles.

Keywords: Chaff; RCS; Turbulence; Modelling and Simulation

\section{Introduction}

Chaff persist from the ancient stages of Second World War. Chaff cloud is cluster of electrical dipoles that resonates at their respective frequency to generate strong backward scattering return for the requisite RCS ${ }^{(1,2)}$. RCS of a target is the measure of the backscattered power per unit solid angle respective to the incident power. Mathematically

$$
\sigma=\lim _{R \rightarrow \infty}\left(4 \pi R^{2} \frac{W s}{W i}\right]
$$


Where $\mathrm{W}_{S}$ and $\mathrm{W}_{i}$ are Scattered and Incident power density respectively; $\sigma$ is the RCS; R is the Range.

In most practical scenario, there is some motion between the target and radar that causes variation with respect to the frequency and the target aspect. This observed RCS is known as Dynamic RCS. Enemy weapon trace various parameter prior to strike and most prolific feature is the RCS of the earmark. When the enemy missile latches the locus of target, it follows the path. To extricate the aircraft from the missile, chaff cloud is being dispensed by the aircraft which mimic as a false target. The RCS of completely bloomed chaff cloud is comparable or greater than the RCS of the deceived object. The aircraft moves away from the missile locked position, leaving only the chaff cloud in its range to which the missile hits ${ }^{(1,2)}$. RCS of chaff clouds is given by

$$
\sigma=\frac{G^{2} \lambda^{2}}{\pi}
$$

where $G$ is the antenna gain.

The fibres are considered to be randomly distributed in three dimensional space with all orientations are equally likely probable. The RCS is averaged over all random orientations with respect to the incident wave. Assuming random orientation of the dipoles and averaging over $4 \pi$,

$$
\sigma=0.172 \lambda^{2}
$$

For $\mathrm{N}$ number of dipoles,

$$
\sigma=0.172 N \lambda^{2}
$$

The chaff designed should fulfill three important features

1) The RCS of the chaff cloud should be remarkable.

2) The chaff cloud should cover the broad range of radar frequency.

3) The blooming time of the chaff cloud should be very less.

The blooming of chaff cloud drive through four phases, first is the birth phase of chaff cloud called transient phase, second is bloom phase in which the formation of chaff cloud begins, third is the Mature phase when the requisite RCS of chaff cloud is attained and last is Decay stage ${ }^{(1,2)}$.

The missile aircraft engagement is very critical in initial few milliseconds. The turbulence can enhance the efficiency of the chaff cartridge in initial engagement and effective break lock. The considerable time required for the blooming and dynamic RCS of chaff fibre must within fraction of second. There are various work has been done related to turbulence. Electromagnetic scattering characteristics of foil in hypersonic plasma turbulence has been reported in ${ }^{(3)}$. Various Cutting Edge Turbulence Simulation methods for wind energy and aerospace problems have been reported in ${ }^{(4)}$. Direct numerical simulation of compressible turbulence in counter flow channel configuration, in which two streams of fluid are brought together from opposite directions is discussed in ${ }^{(5)}$. Bistatic scattering evaluation method for chaff cloud in airflow is presented in ${ }^{(6)}$. $\mathrm{In}^{(7)}$, discrete electromagnetic model for the evaluation of Wideband Bistatic Scattering Responses and statistics of chaff cloud is reported. Modelling and simulation of high speed (rotational) release of chaff cloud is studied and reported in ${ }^{(8)}$. However, in the present paper analysis of turbulence on increasing dispersion efficiency of chaff by varying Turbulent Kinetic Energy (k) is studied and reported in this paper.

\section{Turbulence}

The paramount factor for rapid blooming of chaff cloud is the turbulent kinetic energy due to aircraft movement. In fluid dynamics, the turbulent flow is the pattern of movement in fluid experiencing chaotic changes in the pressure and velocity. In contrast to the laminar flow, there is irregular fluctuation and mixing among the fluids. Turbulent kinetic energy is the mean kinetic energy per unit mass associated in turbulent flows ${ }^{(9)}$. Mathematically, in Computational Fluid dynamics environment the turbulent kinetic energy is

$$
k=\frac{3}{2}(U I)^{2}
$$

Where $\mathrm{U}$ is the initial velocity magnitude; I is the Initial Turbulence Intensity.

$$
I=0.16\left(R_{E}\right)^{\frac{-1}{8}}
$$

https://www.indjst.org/ 
Where $\mathrm{R}_{E}$ is the Reynolds Number.

It is used to indicate whether the fluid flow in term of laminar /turbulent. Higher is the Reynolds number, more is the turbulence in the flow

$$
R_{E}=\frac{\rho v L}{\mu}
$$

Where $\rho$ is the density of fluid; $\mathrm{v}$ is the velocity of fluid with respect to the object; $\mathrm{L}$ is the aircraft wing length; $\mu$ is the dynamic viscosity of fluid ${ }^{(10)}$.

\section{Modelling and Simulation Software}

Ansys Workbench, Ansys Fluent and HFSS-IE have been used to analyse the effect of turbulence on dynamic RCS simulation. ANSYS Workbench is used to define the geometry and meshing properties of primitive over which the operation has to be performed. The flowchart is shown in Figure 1 below.

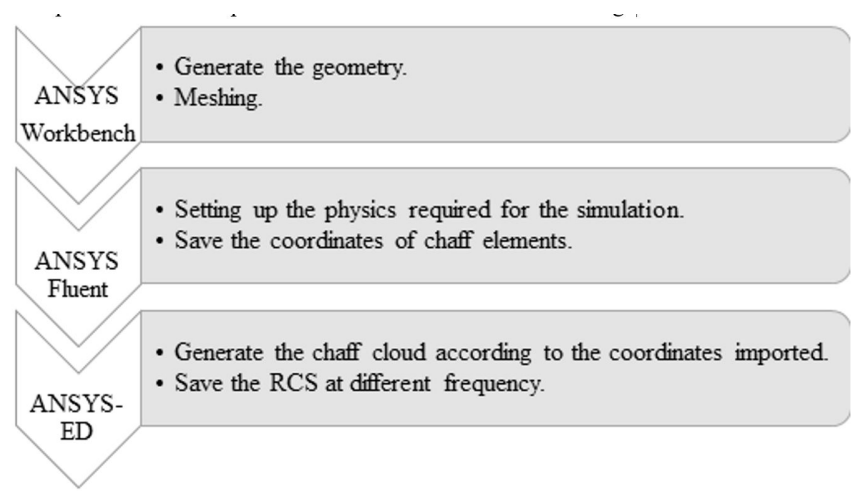

Fig 1. Flowchart of Software

\subsection{Ansys Workbench}

In Design Modeler, the box type primitive is generated and coordinates are assigned to it. In meshing cell, the physics and solver preference is set to CFD and Fluent respectively to create a virtual environment for the blooming of chaff cloud. The hexahedral mesh of proper size is generated over whole geometry and the parts of geometry are defined as inlet, outlet and walls.

\subsection{Ansys Fluent}

The model is then exported to the CFD simulation tool for performing the chaff ejection simulation and obtaining the coordinates of chaff elements at a specific time. The proper physics is set by assigning values to various aerodynamic and materialistic parameters to imitate the real visualization of chaff cloud generation. The energy option is enabled in the setting up physics to activate the heat transfer and temperature dependency. Due to high Mach number, the implicit density-based solver is selected and the gravitation force is applied in $-\mathrm{y}$ direction. The simulations are computed in transient time manner for fixed discrete time steps which are repeated for a number of times till the required duration is obtained. In each time step, the difference solutions between two successive iterations must be around $\mathrm{e}^{-3}$. Since the solver is density based and the flow is not disaggregated, the absolute velocity formulation is preferred and the momentum equations are the function of absolute velocities ${ }^{(10)}$.

The standard $k-\varepsilon$ model with standard near wall treatment is adopted. The inert type elements are injected in group streams in the $y-z$ plane. The velocity of chaff ejection is $35 \mathrm{~m} / \mathrm{s}$ in $\mathrm{z}$-direction while the aircraft movement is in $\mathrm{x}$ direction as shown in Figure 2. 


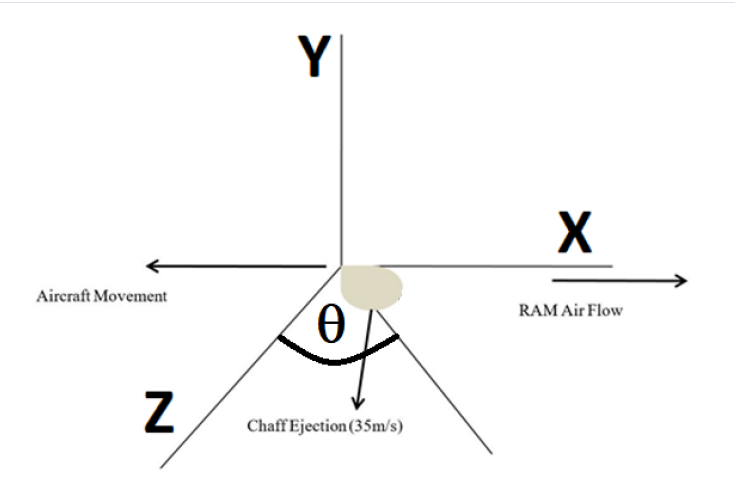

Fig 2. Coordinates systems used for showing Chaff ejection

The effect of turbulent velocity fluctuations on the trajectories of particle is tracked using stochastic tracking by adopting discrete random walk model or eddy lifetime model. The input and output of the simulation box is defined as pressure type, while the discrete phase boundary conditions are defined as escape. The walls of the geometry are provided with the reflect type boundary condition. The particles are reflected in a polynomial manner in both normal and tangential direction. The injections are modeled using non-spherical drag law and the specific shape factor is given according to the diameter of the various chaff element. The pressure acts in the perpendicular direction at input and output box of the model. The standard initialization method is adopted for the field and the initial values are added manually. The first-order transient formulation is selected for the time dependence solution formulations. The spatial discretization for the turbulent related parameters is defined as per first order upwind while for the flow equations, the second-order upwind scheme is selected. The evaluation of gradients is done by adopting least square cell based scheme in which the gradients are calculated between the centroid of one cell to the centroid of another cell. The default values are given to the Courant number and under relaxation factor. After the simulation, the particles are tracked in graphics and coordinates are saved in a file. The Scheme chart for ANSYS Fluent is shown in Figure 3 below.

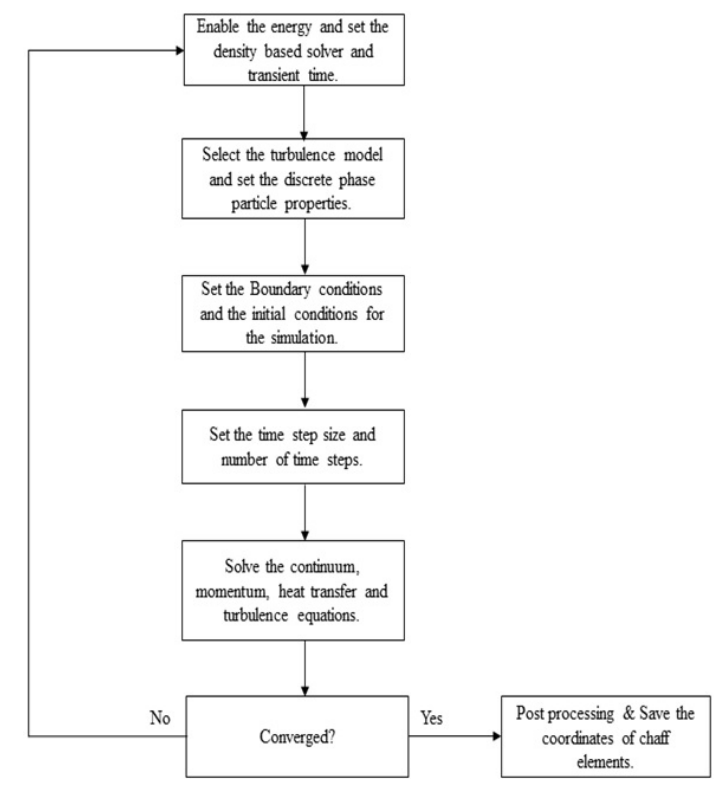

Fig 3. Scheme chart of ANSYS Fluent 


\subsection{Ansys-ED}

HFSS-IE performs surface analysis using Finite Element Method. The python scripts runs in the background to import the excel file of the coordinate position to generate the chaff cloud in HFSS for the calculation of maximum average RCS. The plane incident spherical wave is given as excitation and the infinite sphere far field set up is selected for the calculation of RCS of the chaff cloud. The surface of the chaff fibres is fragmented into number of triangular meshes of lambda refinement $0.15 \lambda$ to calculate current by applying finite conductivity boundary conditions and excitation.

\section{Simulation studies for effect of turbulence on Dynamic RCS of chaff cloud}

A scale down version of chaff clouds is performed by taking 1000 elements of four lengths. These lengths are converted to their equivalent shape factor \& diameter as shown in Table 1 below. To study the subject of paper, simulation was performed for ideal non-turbulent condition compared to turbulent conditions depending upon the angle in which chaff is dispensed from the aircraft.

Table 1. Different electrical filaments taken for RCS analysis

\begin{tabular}{lll}
\hline Equivalent Diameter $(\mu \mathrm{m})$ & Shape factor & No. of filaments \\
\hline 361 & 0.1040 & 250 \\
297 & 0.1260 & 250 \\
278 & 0.1344 & 250 \\
252 & 0.1494 & 250 \\
\hline
\end{tabular}

For evaluation of turbulence, appropriate values of density, air velocity, and viscosity of fluid at heights of $4000 \mathrm{~m}$ were given. As shown in Table 2 below the turbulent kinetic energy varies in the range of $17.5 \sim 18.3 \mathrm{~m}^{2} / \mathrm{s}^{2}$ instretching from parallel to the perpendicular ejection inclination. The transition to turbulent model for boundary air flow occurs when the Reynolds number is greater than $5^{\star} 10^{5}$.

Table 2. Variation of Turbulent Kinetic Energy with different perpendicular ejection inclination

\begin{tabular}{lll}
\hline Angle (degree) & $\mathrm{R}_{E}$ & Turbulent Kinetic Energy k $\left(\mathrm{m}^{2} / \mathrm{s}^{2}\right)$ \\
\hline 0 & $573.087^{\star} 10^{5}$ & 17.5446 \\
30 & $561.649^{\star} 10^{5}$ & 17.729 \\
45 & $548.066^{\star} 10^{5}$ & 17.75 \\
60 & $530.410^{\star} 10^{5}$ & 17.95 \\
90 & $487.733^{\star} 10^{5}$ & 18.375 \\
\hline
\end{tabular}

The cloud expansion with and without turbulent flow at 50ms are shown in Figures 4 and 5 below respectively.

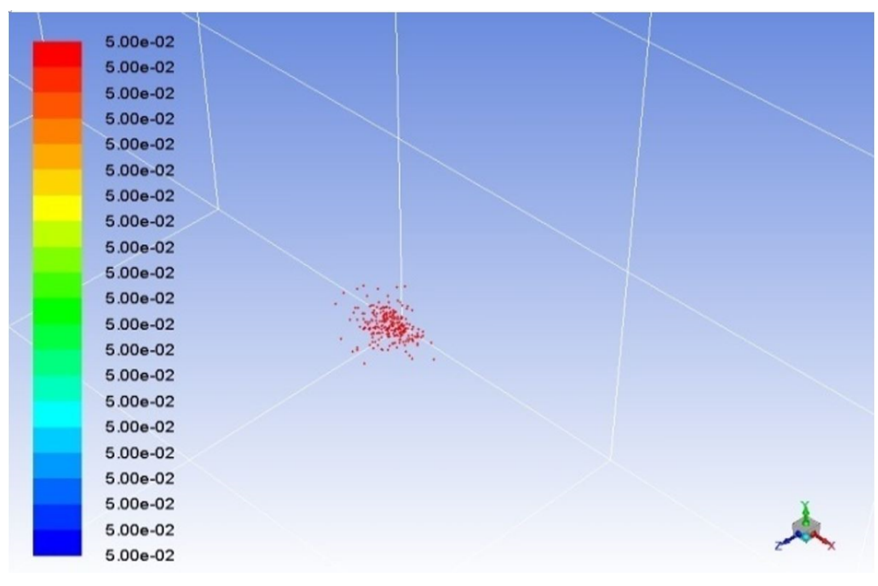

Fig 4. Chaff cloud at $50 \mathrm{msec}$ ( Non-turbulent flow) 


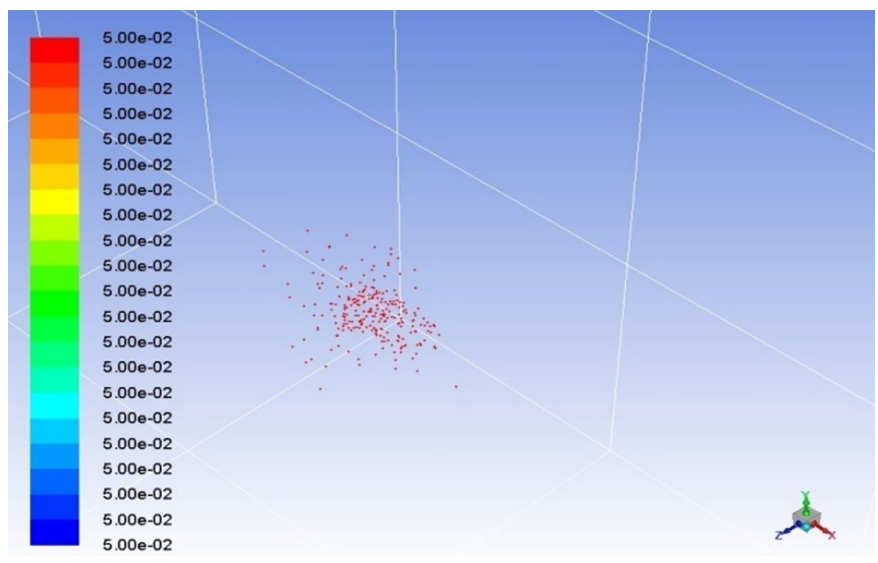

Fig 5. Chaff cloud at $50 \mathrm{msec}$ (Turbulent flow $\mathrm{k}=18.375$ )

\section{Results and Discussion}

There are various previous work that has been reported on turbulence as shown but there is no specific work on effect of turbulence on blooming of chaff cloud when the chaff is fired either parallel of perpendicular ejection. Blooming and dynamic RCS of the 1000 chaff elements was simulated for the time span of $50 \mathrm{msec}$ and $100 \mathrm{msec}$ at different turbulent kinetic energy with ejection angles are shown in Figures 6 and 7 respectively.

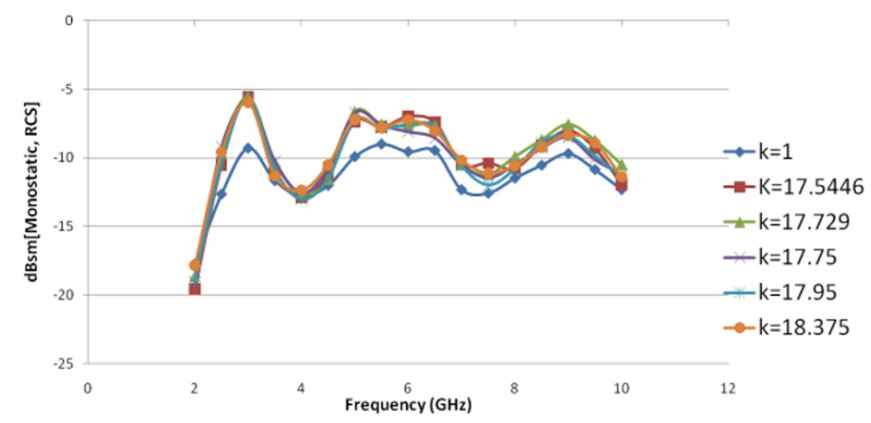

Fig 6. Effect of turbulence on RCS response at $50 \mathrm{msec}$

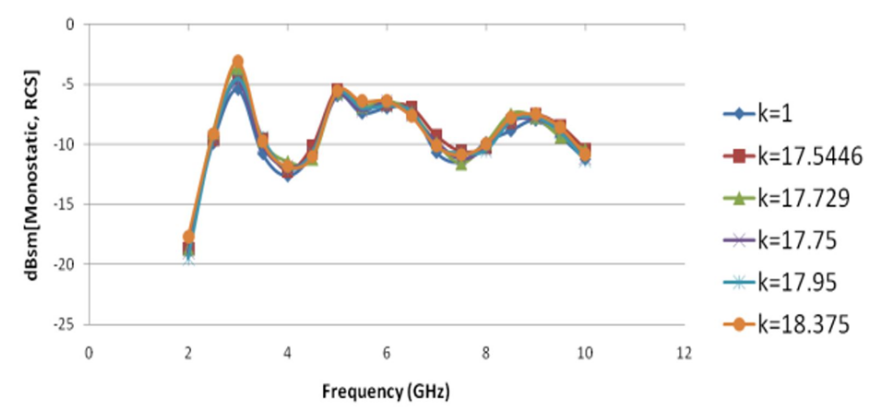

Fig 7. Effect of turbulence on RCS response at $100 \mathrm{msec}$

The RCS of turbulent flow was higher by $1.5 \mathrm{dBsm}$ to $2.5 \mathrm{dBsm}$ for $\mathrm{t}=50 \mathrm{msec}$. The RCS response of non-turbulent model at $\mathrm{t}$ $=100 \mathrm{msec}$ become almost identical to the turbulent model RCS response at $\mathrm{t}=50 \mathrm{msec}$, with slight divergence of around \pm 0.5 $\mathrm{dBsm}$. It can be perceived that the turbulence effect has reduced the blooming time of chaff filaments in the range of $25 \mathrm{msec}-$ $50 \mathrm{msec}$. While collating the results between turbulence for perpendicular ejection of chaff fibres $\left(\theta=90^{\circ}\right)$ and parallel ejection 
of chaff fibres $\left(\theta=0^{\circ}\right)$, it is observed that initially at $t=100 \mathrm{msec}$, RCS response of former was higher than the latter by $0.1 \mathrm{dBsm}$ $1 \mathrm{dBsm}$. After a certain time both the cases had almost similar responses, it can be sensed from the above fact that the turbulence has greater effect for perpendicular ejection.

\section{Conclusion}

The turbulence effect has reduced the blooming time of chaff filaments in the range of $25 \mathrm{msec}-50 \mathrm{msec}$. It can be observed from figure 7 that the RCS of non-turbulent $(\mathrm{k}=1)$ chaff cloud becomes equal to turbulent chaff $(\mathrm{k}>1)$ cloud RCS at $100 \mathrm{msec}$. However, when we observe and compare RCS of non-turbulent $(k=1)$ chaff cloud and turbulent $(k>1)$ chaff cloud shown in figure 6 with figure 7, it can be observed that the RCS of non-turbulent chaff cloud that we get at $100 \mathrm{msec}$ could be easily get at 50 msec turbulent $(k>1)$ chaff cloud. It can be inferred that the turbulence has a pivotal role in dispersal of chaff filaments resulting in surge in inter dipole spacing and RCS values of chaff during initial firing. Original chaff cartridges consist of millions of chaff filaments, this analysis can be further extrapolated to simulate the effect of turbulence in a practical case scenario.

\section{References}

1) Kumar V, Vasistha P, Kumar R. RCS Simulation and Measurement Studies of Microwave Dipoles for Chaff Applications. International Journal of Advances in Microwave Technology. 2019;04(01):185-189. Available from: https://dx.doi.org/10.32452/ijamt.2019.185189. doi:10.32452/ijamt.2019.185189.

2) Kumar V, Singh AK, Vasistha P, Kumar R. Dynamic RCS Prediction of Rapidly Blooming Chaff Cloud and its Validation using Measurement on Scaleddown. International Journal of Advances in Microwave Technology. 2018;3(4):170-175. Available from: https://dx.doi.org/10.32452/ijamt.2018.170175. doi:10.32452/ijamt.2018.170175.

3) Jiangting L, Teng G, Lixin G. Electromagnetic scattering characteristics of foil in hypersonic plasma turbulence. IET Microwaves, Antennas \& Propagation. 2019;13(14):2575-2579. Available from: https://dx.doi.org/10.1049/iet-map.2018.5310. doi:10.1049/iet-map.2018.5310.

4) Heinz S, Peinke J, Stoevesandt B. Cutting-Edge Turbulence Simulation Methods for Wind Energy and Aerospace Problems. Fluids. 2021;6(8):288-288. Available from: https://dx.doi.org/10.3390/fluids6080288. doi:10.3390/fluids6080288.

5) Hamzehloo A, Lusher DJ, Laizet S, Sandham ND. Direct numerical simulation of compressible turbulence in a counter-flow channel configuration. Physical Review Fluids. 2021;6(9):2575-2579. Available from: https://dx.doi.org/10.1103/physrevfluids.6.094603. doi:10.1103/physrevfluids.6.094603.

6) Zuo Y, Guo L, Liu W, Liu S. A Bistatic Scattering Evaluation Method of the Chaff Cloud in Airflow Based on VRT. In: and others, editor. IEEE Transaction on Antennas and Propagation. 2021. doi:10.1109/TAP.2021.3090813.

7) Alvarez J, Bocanegra DE. Discrete Electromagnetic Model for the Evaluation of Wideband Bistatic Scattering Responses and Statistics of Chaff Clouds. IEEE Transactions on Antennas and Propagation. 2020;68(8):6256-6264. Available from: https://dx.doi.org/10.1109/tap.2020.2985771. doi:10.1109/tap.2020.2985771.

8) Tong J, Liu Z, Xu L. The Modeling and Simulation of Chaff Release at High Speed. Journal of Physics: Conference Series. 2021;1813(1):012001-012001. Available from: https://dx.doi.org/10.1088/1742-6596/1813/1/012001. doi:10.1088/1742-6596/1813/1/012001.

9) Batchelor GK. Flow at Large Reynolds Number: Effects of Viscosity. In: and others, editor. An Introduction to fluid dynamics. ;p. $264-377$. doi:10.1017/СBO9780511800955.007,264-377.

10) Kashyap R, Kumar V, Gangwar RK, Vasistha P, Kumar R. RCS Analysis of scaled down chaff clouds using Ansys ED(HFSS) to understand the behaviour of real time model. 2018 IEEE MTT-S International Microwave and RF Conference (IMaRC). 2018. 